\title{
Solar Panel Tracking System Using Optical Fiber
}

\author{
Lorant Andras Szolga ${ }^{1, *}$, and Zsolt Farnas ${ }^{1}$ \\ ${ }^{1}$ Faculty of Electronics, Telecommunications and Information Technology, UTCN, Basis of Electronics Department, Optoelectronics \\ Group, Cluj-Napoca, Romania
}

\begin{abstract}
In this work we present a new method for the measurement of the light intensity for the solar panels with dual axis tracking system by using optical fiber to conduct the direct sunlight to the light dependent resistors (LDR). With this method we keep away the influence of the temperature on the photoresistors which measure the light intensity. The measurements with the proposed system was done at the same time and place with a classical system with the same configuration, but with the LDRs directly exposed to the sunlight. The results highlighted that, in cloudy conditions when the sunlight is not in direct path on the solar panels and suffers a high scattering, the optical fiber system is more efficient compared to the classical one with differences up to $18 \%$ in the generated power.
\end{abstract}

\section{State of the art}

In the last years we are witnesses to the climatic changes caused by the high pollution of the fossil fuels. The governments all over the world are supporting research in the field of renewable energies. Among this energy is the solar energy.

The Sun is an incredible source of energy, which emits around $1.75 \cdot 10^{17} \mathrm{~J} / \mathrm{s}$ on the Earth surface. The energy radiated by the Sun to the Earth during an hour could cover the energy necessary for our planet for a whole year. A brief analysis showed that only fifteen minutes of the Sun's energy would cover in $100 \%$ the annual energy necessities for a year for Great Britain [1]. For the moment, the $100 \%$ conversion of this energy is not a reasonable objective. However, using photovoltaic cells the solar energy can be transformed into electrical energy that serves our daily energy use. To take the maxim benefits that the actual technology can offer in this field we should understand the mechanism of solar energy capture. In general, the solar cells collect the direct solar radiations, but to get the maximum efficiency out of it the radiations must be perpendicular on the cells. Because the Earth spins around the Sun and its own axis, the spatial position of the Earth is in continuous change, which means that if the solar cells have a fixed position the day light will be perpendicular to the solar cells for a short amount of time. It was proven that for the fixed solar cells the maximum reachable efficiency is $14 \%$ [2]. More than that it requires a careful mounting for the best angle to obtain a reasonable efficiency [3-4]. To raise the productivity, the solar panels should be oriented in a manner that the light reach them perpendicularly. To do this an automated positioning system should be used to follow closely [5].

One solution in this direction is the passive tracking system where the rotation movement is done by thermal expansion using two cylinders filled with fluids under pressure (Freon). When one of the cylinders is exposed to the light the pressure in that cylinder will raise. To keep the equilibrium a part of the liquid flows to the cylinder being in the shadow and thus the attached actuators to the cylinders will move the panel. This type of trackers is not very precise because can be affected very easily by the wind [6].

Another solution is by using active tracking systems, with one axis or two axes, which are using motors to orient the panels after the direction of the Sun. This type of systems is more accurate and efficient than the passive systems. The active tracking system with one axis will follow the Sun only from East to West. The disadvantage is the fixed angle from the base of the system, which cannot compensate the yearly movement of the Sun by a tilt angle of 23.5 degrees as it moves in an east-west direction. Thus, there are restricted not to follow the movement of the Sun for a perpendicular radiation in different seasons of the year [7]. Despite of this limitation these types of systems are capable to raise the captured power with $35 \%$ as against the fix photovoltaic panels [8].

The bidirectional tracking system can be considered the best one, by following the Sun movement in a manner to have always perpendicular radiations on the panel during all the days of a year (Fig.1). This leads to an optimized power. Compared to the best fixed panel system (the one that uses the optimum monthly angle) the dual axis system can produce $43.87 \%$ more energy [6].

A complex analysis of the effect of the clouds on the solar radiations reaching the surface of the Earth is presented in [9], where the difference between a clear sunny day and a cloudy day can reach up to $700 \mathrm{~W} / \mathrm{m}^{2}$.

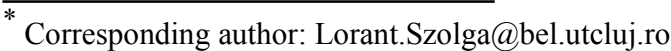




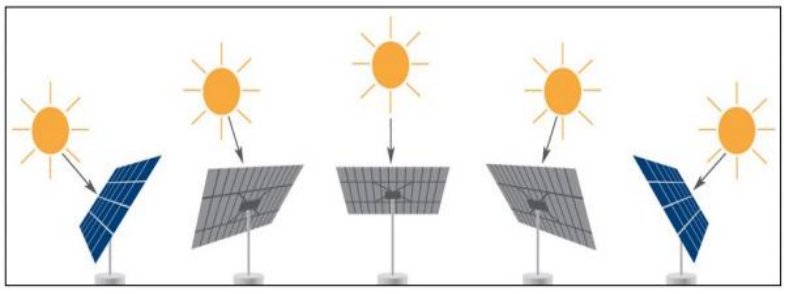

Fig. 1. Dual-axis tracking system [C. Juda, 2013].

\section{Hardware configurations}

To develop a dual-axis tracking system, we need to detect the light intensity. The best widespread techniques to detect the light intensity is with light dependent resistors (LDR) whose resistance decreases with the increase of the light intensity. For the implemented systems we used solar panels with the following manufacturer specifications: maximum output voltage $6 \mathrm{~V}$, maximum output current $200 \mathrm{~mA}$ and maximum generated power of $1.2 \mathrm{~W}$. For this type of panels, we decided that two $12 \mathrm{~V}$ servo motors are more than enough to serve their movement on a two-axis system. The LDR detection and servo motors control was processed by an ATmega328 microcontroller with an easy programming on an Arduino Uno development board (Fig.2). From the electronics point of view the two tested system is the same. We will call the system where the LDRs are exposed directly to the sunlight as classic tracking system and the new one proposed by us as optical fiber tracking system.

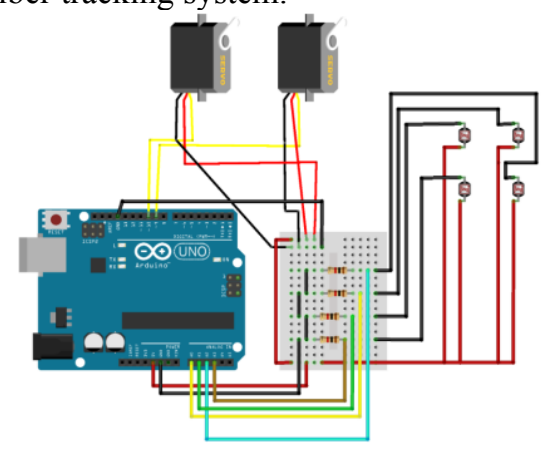

Fig. 2. Arduino Uno based tracking control system.

\subsection{Dual axis classic tracking system}

As mentioned before one of the systems will use the LDRs in a direct sunlight (Fig.3), so the LDRs will be mounted on the top side of solar panel where there are separated from each other by a $3.5 \mathrm{~cm}$ wall (Fig.4). The scope of this wall is to create shadow on the LDRs when the sunlight is not perpendicular on them. We printed out the shadowing mask in PLA material which is highly environmentally friendly. Ideally at constant light intensity, the resistance of a photoresistor should remain constant, Practically, the resistance of LDRs changes non-linearly to the intensity of light. More than that for constant light intensity the resistance may still fall due to temperature changes. To get rid of this issue as much as we can, we used a white PLA which will reflect the light and won't overheat the LDRs assembly.

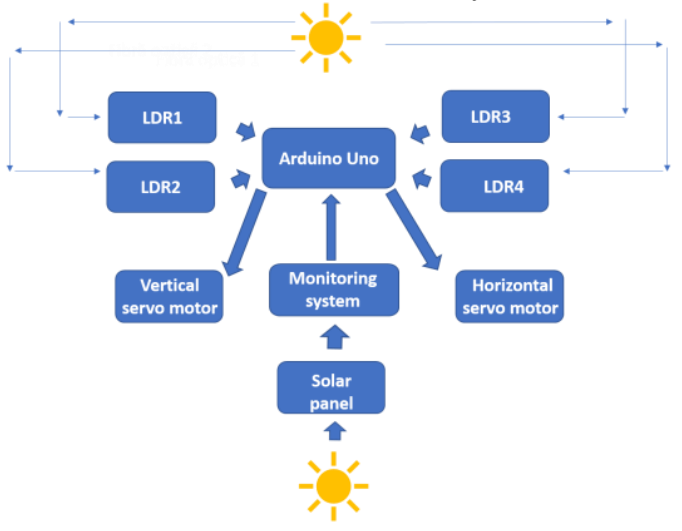

Fig. 3. Block diagram of the dual-axis classic tracking system.

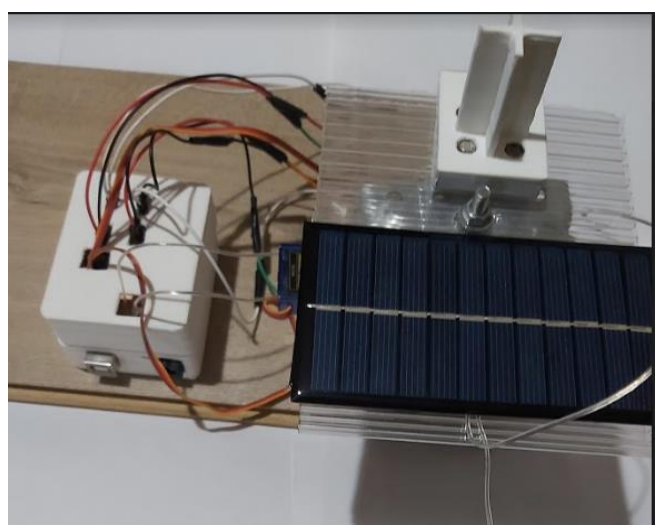

Fig. 4. Implemented dual axis classic tracking system.

\subsection{Dual axis optical fiber tracking system}

For the second system, the light intensity is received by each LDR through a $1 \mathrm{~m}$ long plastic optical fiber (POF) with a typical core diameter of $0.98 \mathrm{~mm}$ (Fig.5). One end of the fibers is placed on top of the solar panel and separated by a $3.5 \mathrm{~cm}$ wall and the other end is connected in the control box (Fig.6) where the LDRs are attached to the electronic shield of the Arduino Uno board. In this manner the light is conducted to the LDRs by the optical fiber and the LDRs are protected from overheating.

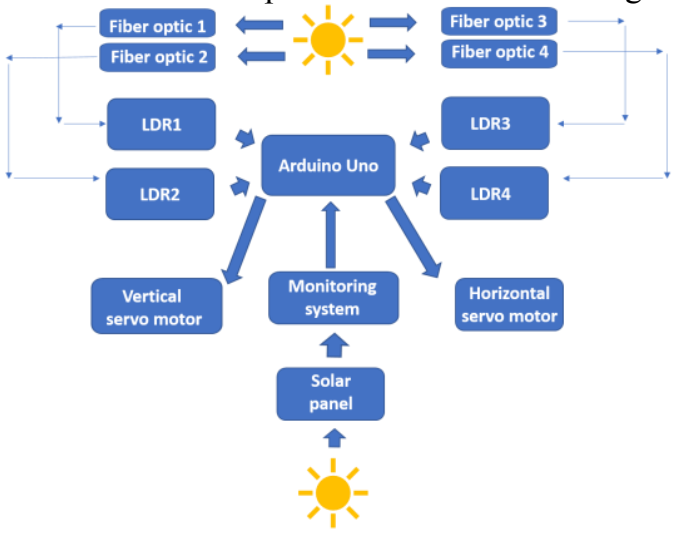

Fig. 5. Block diagram of the dual axis optical fiber tracking system. 


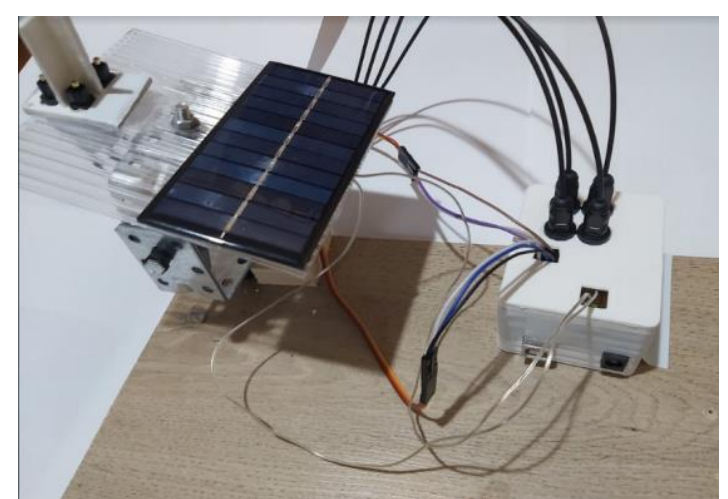

Fig. 6. Implemented dual axis optical fiber tracking system.

\subsection{Control loop}

The measurement of the light intensity and the movement of the servo motors are loaded into the ATmega328 microcontroller. The four LDRs are grouped in the control loop (Fig.7) as follows: the top pair and the bottom pair monitor the light intensity from east to west; the left pair and the right pair monitor the deviation angle due to the seasonal position change.
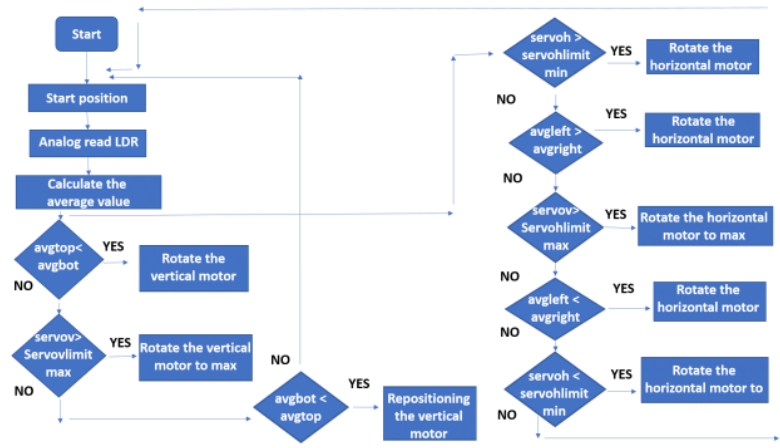

Fig. 7. Logic diagram of the controller program.

\subsection{Voltage and current monitoring}

For the continuous measurement of the electrical power generated by the two systems we measured the voltage and the current generated by them. In both cases a simple voltage divider system was implemented, where the voltage of interest was measured by one of the analog inputs of the microcontroller. In a simple test of the solar panels in direct sunlight we measured an open circuit voltage of $6.4 \mathrm{~V}$, instead of $6 \mathrm{~V}$ from the manufacturer specifications. Thus, we made all the further calculus by taking the maximum voltage developed by the solar cells $6.4 \mathrm{~V}$. In the case of the solar panel's voltage monitoring (Fig.8) the voltage divider will scale down the voltage under $5 \mathrm{~V}$ which is the maximum value accepted by the microcontroller ADC converter:

$$
V_{A D C}=\left[R_{2} /\left(R_{1}+R_{2}\right)\right] * V_{\text {panel }}
$$

Choosing the resistors properly $\left(\mathrm{R}_{1}=15 \mathrm{k} \Omega\right.$, $\mathrm{R}_{2}=47 \mathrm{k} \Omega$ ) the maximum voltage read by the ADC is $4.85 \mathrm{~V}$.
In the case of the solar panel's current monitoring the voltage was read on the shunt resistor $\mathrm{R}_{4}=1 \Omega$ (Fig.8):

$$
V_{A D C}=\left[R_{4} /\left(R_{3}+R_{4}\right)\right] * V_{\text {panel }}
$$

With a value of $\mathrm{R}_{3}=30 \Omega$ the maximum measured current is $197 \mathrm{~mA}$. Using the internal reference of $1.1 \mathrm{~V}$ of the microcontroller we could measure currents low as $1 \mathrm{~mA}$ :

$$
I_{\text {min }}=V_{r e f} /\left(1024 * R_{4}\right)
$$

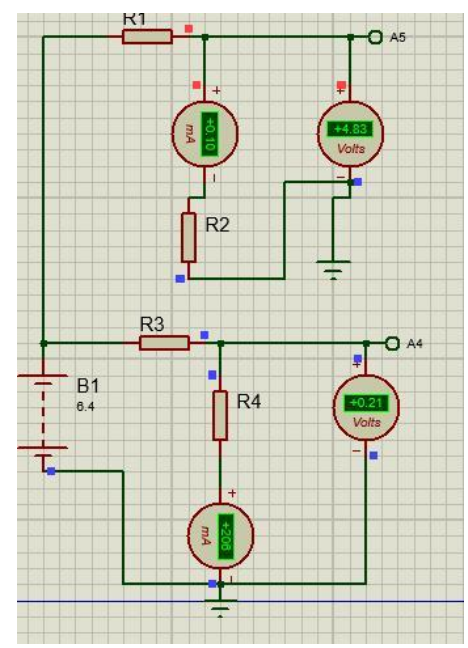

Fig. 8. Voltage and current measurement circuits.

\section{Measurements}

The measurements where conducted, with the two implemented systems mounted in the same area, for more days in a raw. The data points of the voltage and current produced by the two systems where analysed and the developed power was calculated. From all this data sets we are showing the results obtained on two days when the weather was changing from a clear sunny sky to a cloudy one. The data plots of these days are presented as follows: current in Fig.9, Fig.12 and Fig.15; the voltage in Fig.10, Fig.13 and Fig16; the power in Fig.11, Fig.14 and Fig. 17.

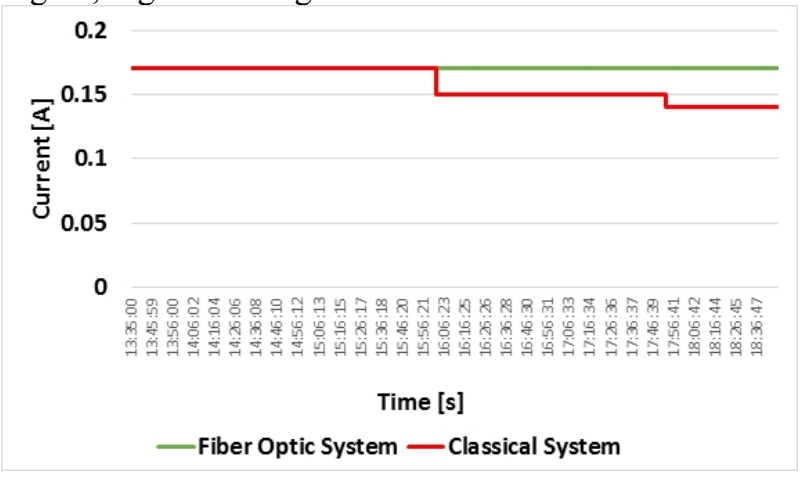

Fig. 9. Current measurement Day 1. 


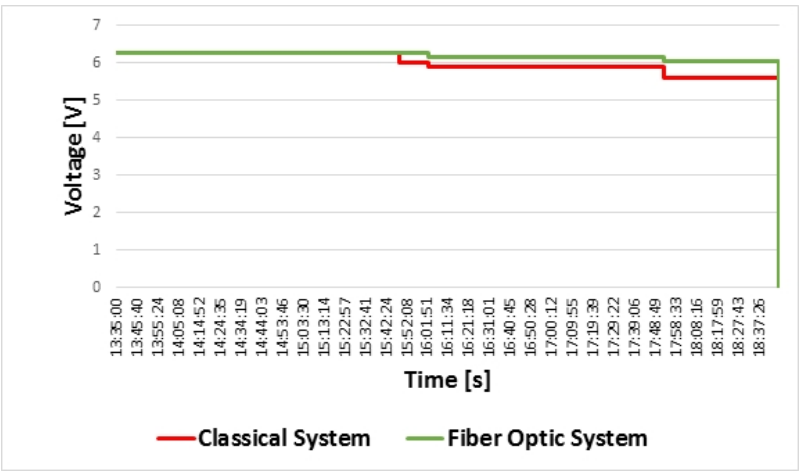

Fig. 10. Voltage measurement Day 1.

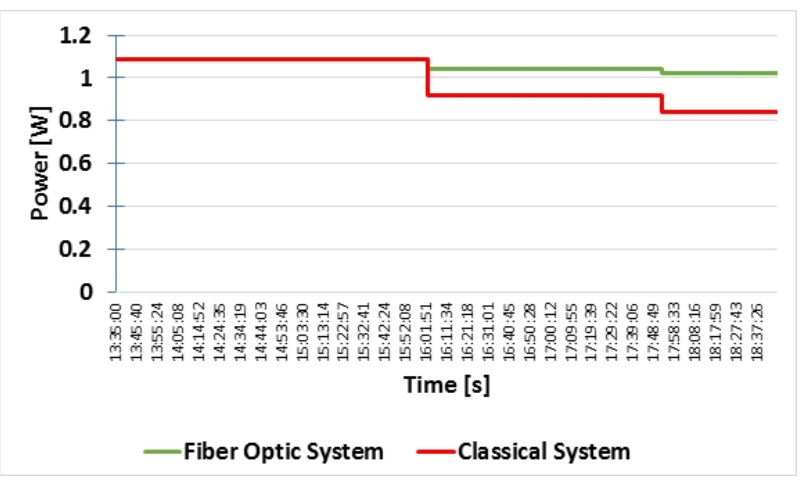

Fig. 11. Power measurement Day 1.

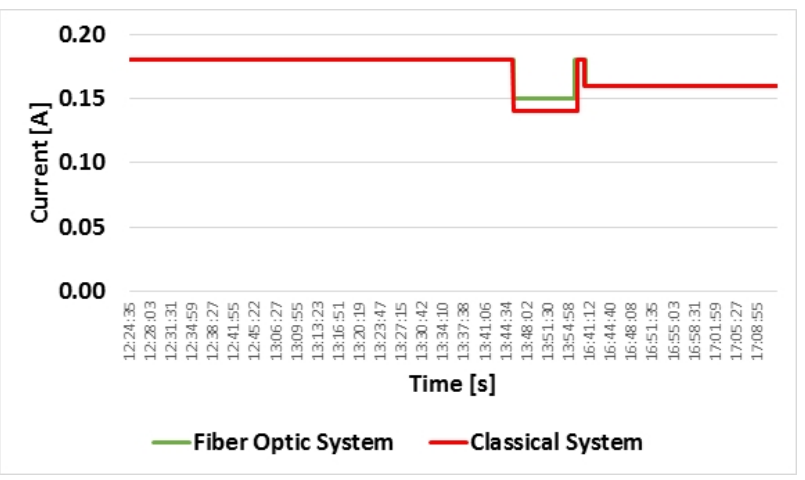

Fig. 12. Current measurement Day 2.

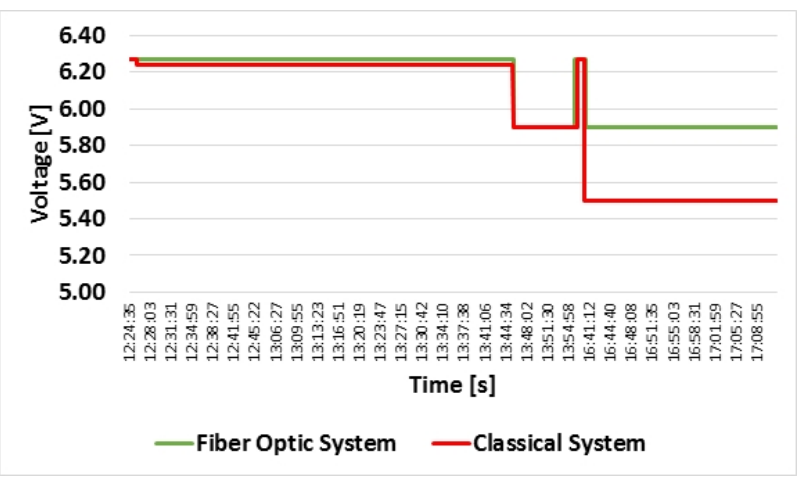

Fig. 13. Voltage measurement Day 2.

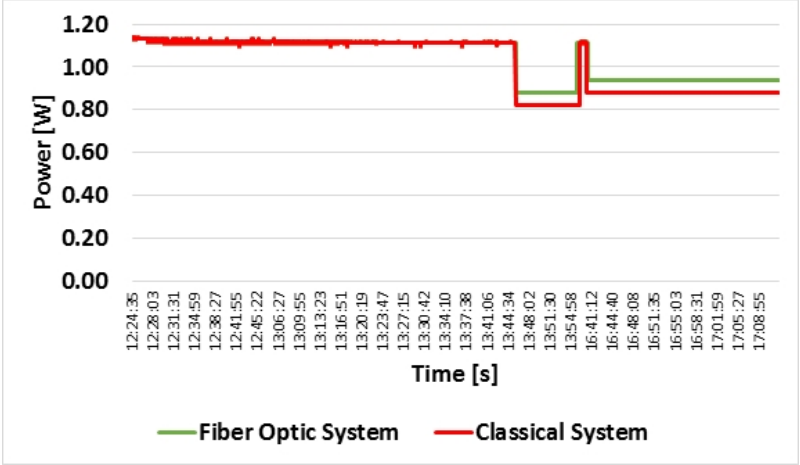

Fig. 14. Power measurement Day 2.

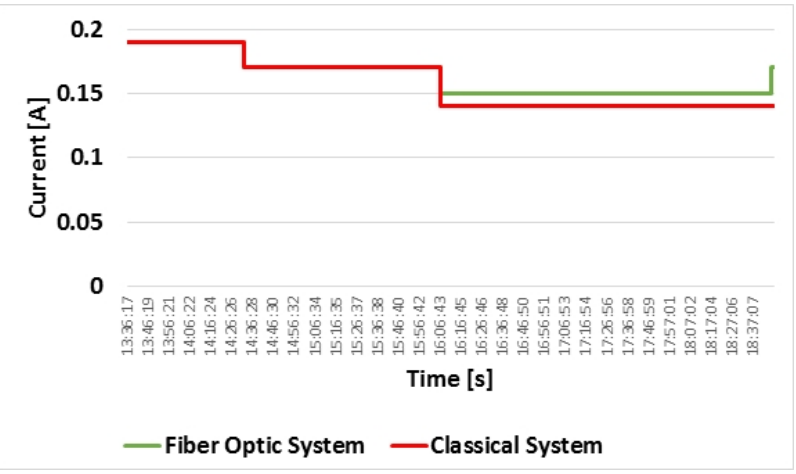

Fig. 15. Current measurement Day 3.

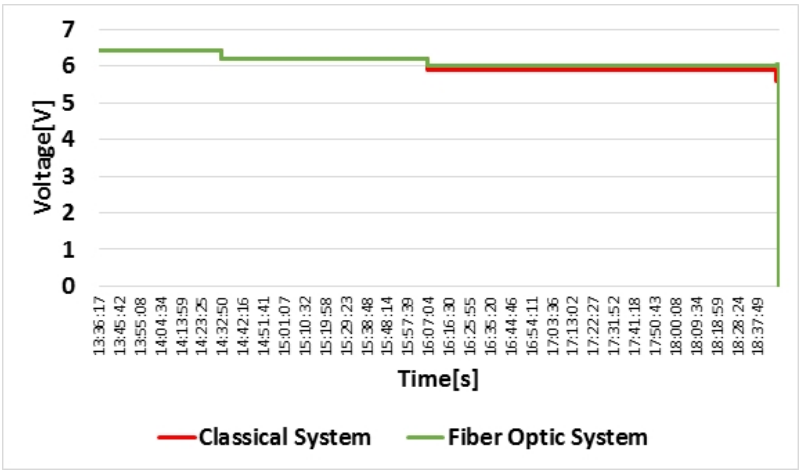

Fig. 16. Voltage measurement Day 3.

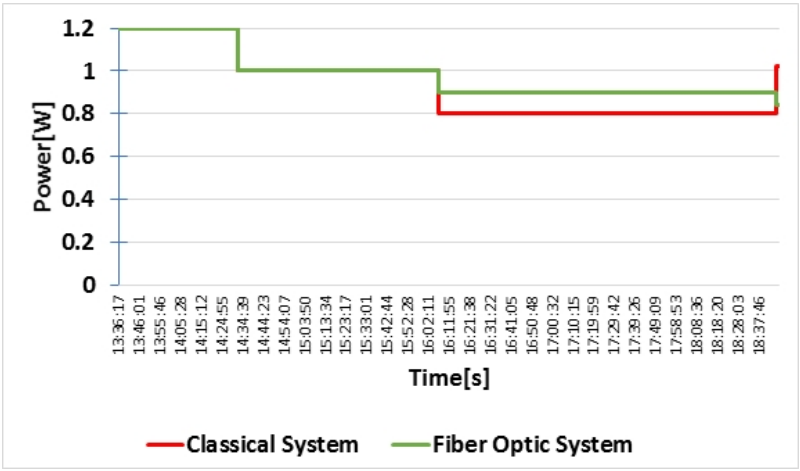

Fig. 17. Power measurement Day 3. 
We could observe that at noon when we had a clear sky and a lot of sunshine the two systems developed the same voltage $(6.3 \mathrm{~V})$ and current $(180 \mathrm{~mA})$ and resulted in the same generated power $(1.1 \mathrm{~W})$. In the afternoon when we had a cloudy sky and we couldn't see the sun, the systems presented a lower voltage and current, values that had a direct influence on the generated power. In all these cloudy conditions the measurements of the voltage and current for the optical fiber tracking system was better that the classic tracking system. To have a better comparison on these power values we centralized them in Table 1, where for the cloudy sky conditions we placed the lowest value that the systems measured.

Table 1. Power generated by the two systems in different days and weather conditions.

\begin{tabular}{|c|c|c|c|c|}
\hline \multirow{2}{*}{ Day } & \multicolumn{2}{|c|}{$\begin{array}{c}\text { Generated Power } \\
\text { with Classic System } \\
\text { [W] }\end{array}$} & \multicolumn{2}{|c|}{$\begin{array}{c}\text { Generated Power with } \\
\text { Optical Fiber System } \\
\text { [W] }\end{array}$} \\
\cline { 2 - 5 } & $\begin{array}{c}\text { Clear } \\
\text { Sky }\end{array}$ & $\begin{array}{c}\text { Cloudy } \\
\text { Sky }\end{array}$ & $\begin{array}{c}\text { Clear } \\
\text { Sky }\end{array}$ & $\begin{array}{c}\text { Cloudy } \\
\text { Sky }\end{array}$ \\
\hline 1 & 1.1 & 0.8 & 1.1 & 1 \\
\hline 2 & 1.1 & 0.8 & 1.1 & 0.87 \\
\hline 3 & 1.1 & 0.8 & 1.1 & 0.9 \\
\hline
\end{tabular}

\section{Conclusions}

We started out the presented work by analyzing the influence of the temperature on the LDRs used in the dual axis tracking systems of the solar panels. We could observe that isolating the LDRs and conducting the sunlight through them by optical fiber don't have a significant effect on the tracking system when we have a clear sunny sky. In these conditions we obtained the same maximum generated power of $1.1 \mathrm{~W}$. It means that the temperature doesn't affect the measurements conducted by the LDRs. Instead, in cloudy conditions the optical fiber tracking system proved to be better than its classical counterpart. From the data analysis, where the reference point is the maximum power generated by the panels $(1.1 \mathrm{~W})$, in the case of the classic tracking system in cloudy conditions we will get almost $73 \%$ of power $(0.8 \mathrm{~W})$. On the other hand, in the same weather conditions, with the optical fiber tracking system we could get in the worst case only $79 \%$ of power $(0.87 \mathrm{~W})$ and in the best scenario of $91 \%$ of power $(1 \mathrm{~W})$. Comparing the two tracking systems the increase of the power generated by the optical fiber system over the power generated by the classic system can reach up from $6 \%$ to $18 \%$. We consider this a big benefit in the solar energy harvesting systems.

\section{References}

1. I. Dincer, A.S. Joshi, Solar Based Hydrogen Production Systems (Springer Science, 2013)

2. M. Gürtürk, UEMK, (2019)

3. A. McEvoy, L. Castaner, Tom Markvart, Solar Cells, (Elsevier 2013)

4. J.E. Braun, J.C Mitchell, Solar Energy, 31 (1983)

5. A.M. Catarius, M.P. Christiner, Dig. WPI, (2010)

6. M. Kacira, S. Sadanori, O. Kacira, O. Limi, M. Ishi, H. Kowata, H. Moriyama, J. of Agric. Meteorol. 60, (2004)

7. Y. Suita, S. Tadakuma, Int. Conf. on Ind. Tech., (2006)

8. H. Huang, J. Huang, Organic and Hybrid Solar Cells (Springer, 2014)

9. D. Matuszko, Int. J. Of Clim. 32 (2012) 